\title{
Pelvic Textiloma Mimicking a Hydatid Cyst: A Case Report
}

\author{
Ouha $\mathrm{M}^{1 *}$, kyabaambu Wumba Kilonda $\mathrm{CM}^{1}$, Ranib $\mathrm{M}^{1}$, Boutakiout $\mathrm{B}^{1}$, Ouali Idrissi $\mathrm{M}^{1}$, Cherif Idrissi El Ganouni $\mathrm{N}^{1}$
}

Radiology Department, ARRAZI Hospital, University Hospital Mohamed VI, Marrakech, Morocco

DOI: $10.36347 /$ sasjm.2021.v07i04.008

| Received: 18.03.2021 | Accepted: 23.04.2021 | Published: 29.04.2021

*Corresponding author: M. Ouha

Abstract

Case Report

Textiloma, is a rar but well-known postoperative complication. We report the case of a 39 year-old female patient operated 5 years for an ovarian cyst. The postoperative course was uneventful until 2019 when she presented a bowl obstruction. Investigations revealed a pelvic csyst which was thought to be a hydatid cyst, but operative findings showed textiloma.

Keywords: Textiloma, bowl obstruction, hydatid cyst.

Copyright $(\mathcal{C} 2021$ The Author(s): This is an open-access article distributed under the terms of the Creative Commons Attribution 4.0 International License (CC BY-NC 4.0) which permits unrestricted use, distribution, and reproduction in any medium for non-commercial use provided the original author and source are credited.

\section{INTRODUCTION}

Textiloma, also called "gossybipoma", is a rare but well-known postoperative complication. It is used to describe a foreign body made up of compress (s) or surgical drape (s) found at a surgical site [1]. With a frequency of $22 \%$, gynecological textilomas represent the second most common textilomas after intraperitoneal textilomas (52\%) [2]. Due to its varied clinical and radiological presentations, textiloma can be confused with several focal lesions, thus representing a diagnostic dilemma [3]; also a precise history, combined with clinical and radiological data are essential to suspect its diagnosis. Thus we report the observation of a 39-year-old patient with a history of pelvic surgery for an ovarian cyst, in which the diagnosis of a pelvic textiloma, whose appearance was similar to that of a hydatid cyst, was made.

\section{OBSERVATION}

A 39-year-old patient had been urgently admitted for a non-febrile intestinal occlusion developing for 3 days. This syndrome consisted of abdominal distension, Inability to have a bowel and gas and vomiting without other associated clinical signs. The patient had a history of pelvic surgery for an ovarian cyst dating from 5 years before her admission but without documents on the pathology and the nature of the surgery performed.
After conditioning, an unprepared abdominal $\mathrm{x}$-ray (ASP) was performed showing larger than high hydro-aeric levels pointing to a hail-like digestive occlusion (Figure 1).

Considering the aspect of the abdominal plain radiograph, an abdominal computed tomography (CT) with and without injection of contrast was carried out subsequently revealing a distension of the intestinal loops with or without the presence of fluid levels. CT also showed a voluminous pelvic cystic formation measuring 7.5 x 10 × $8.5 \mathrm{~cm}$, thin-walled, with hypodense content, site of multiple floating hyperdense membranes, unmodified after injection of the contrast. Large bowl was flattened and there were no signs of complications (Figure 2).

With this tomodensitometric aspect of a thinwalled cystic formation on which there are floating membranes, not enhanced by contrast and, the diagnosis of pelvic hydatid cyst was in the top of the list.

Surgical exploration found an $8 \times 7 \mathrm{~cm}$ mass surrounded by the great omentum, containing a purulent collection plus a $10 \times 7 \mathrm{~cm}$ surgical pack, adherent to the sigmoid wall and to the intestins, $60 \mathrm{~cm}$ from the ileocecal junction. The postoperative course was unremarkable. 


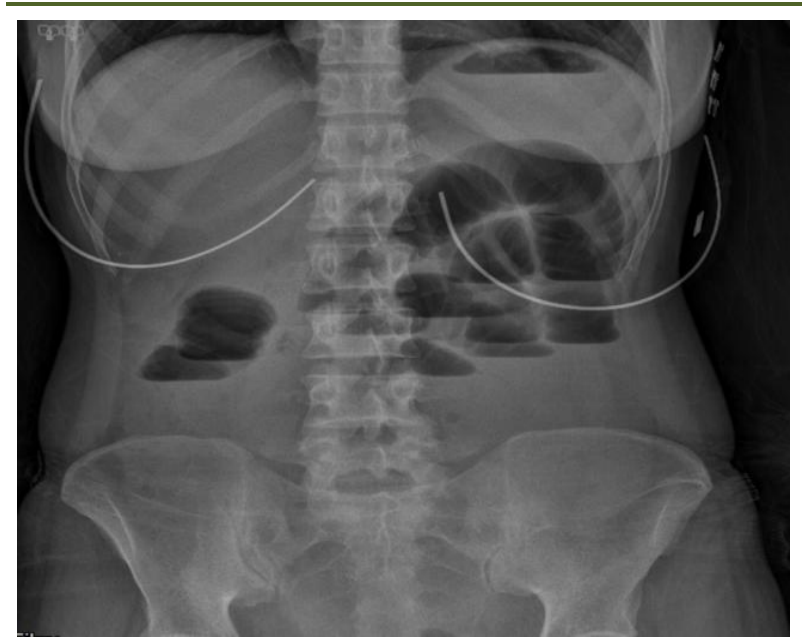

Fig-1: Abdominal plain radiograph in standing position showing wider than high fluid levels

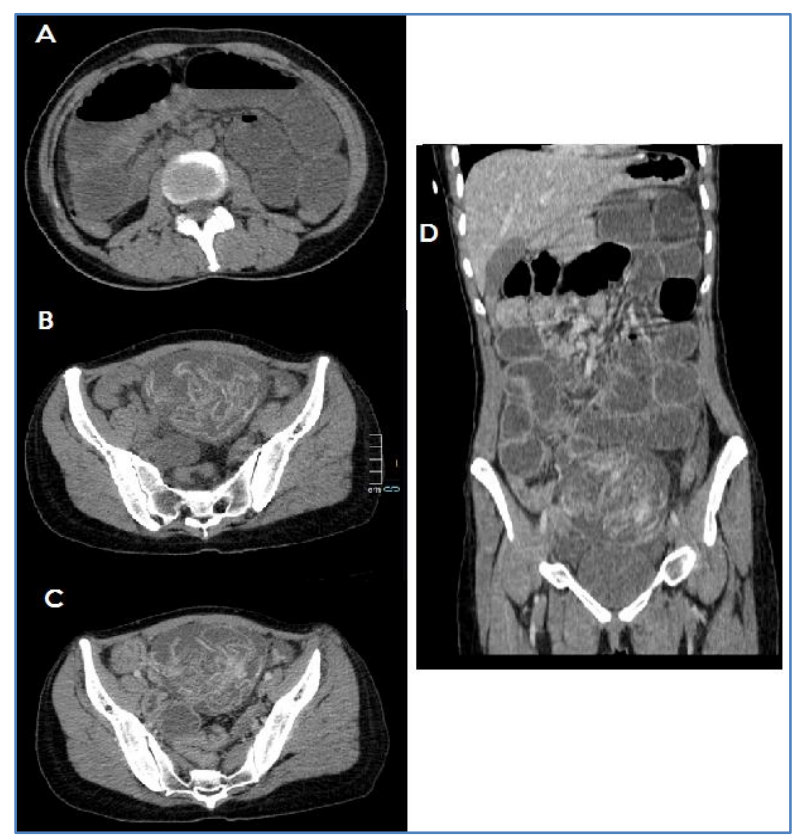

Fig-2: Abdominal CT in axial slices without $(\mathrm{A}, \mathrm{B})$ and after injection (C) and coronal reconstruction (D).

Presence of fluid levels (A) and presence in the pelvic area of a large pelvic cystic formation, thin-walled, hypodense content, seat of floating membranes, spontaneously hyperdense, unmodified after injection of PDC

\section{DISCUSSION}

Textilomas can physiopathologically cause two types of reactions: a fibroblastic reaction, such as a foreign body reaction or an exudative reaction which often leads to an abscess (pain, fever, weight loss, and fistula). The second reaction can also lead to intestinal obstruction or perforation, caused by surgical adhesions, granulomatous peritonitis and sepsis [3, 4].

It can be discovered in the first few days after surgery or can go asymptomatic for many years and be discovered accidentally. In our case, the surgical pads left in the patient's body did not produce any symptoms for five years, this substantially corroborates with the data in the literature which report an average time of detection of 6 to 9 years [5].

The clinical presentation of gossybipoma is variable, depending on its location and the type of biological reaction [6]. In our case, it was a bowl obstruction.

Different radiological aspects have been described in the literature [7]; and cases of textiloma simulating a hydatid cyst have been reported but in other locations: pericardial [8] thoracic [9] abdominal $[2,10]$.

The diagnosis of textiloma can be suggested by a simple abdomino-pelvic ultrasound which shows a well-limited fluid formation containing hyperechoic, serpiginous and striped structures, generating a posterior shadow cone: These structures correspond to the forgotten textiloma $[7,10]$. In our observation, the ultrasound was not performed due to the urgency of the bowl obstruction.

CT allows a precise preoperative topographic diagnosis and performs a complete exploration of the abdominal cavity in search of associated postoperative complications. It shows suggestive aspects related to a foreign body: a spongiform appearance due to gas trapped in the tissue, or a heterogeneous appearance with hyperdense banded or hypodense vortex structures. One or more calcifications can be seen $[7,8,10]$. The latter aspect being similar to hydatid cysts as reported in our case.

The diagnosis of textiloma must be systematically evoked in an operated patient. The confirmation of this diagnosis remains however surgical. Also in a retrospective study reporting eight cases of thoracic textiloma. Ridene et al. report that the diagnosis was confirmed intraoperatively in all cases [9]. Confirmation of this diagnosis intraoperatively was also the case in our presentation.

\section{CONCLUSION}

Textiloma is a fairly frequent complication of abdominal and pelvic surgeries and must be systematically mentioned even in the face of nonspecific aspects. The discovery of a textiloma is recognized as a serious fault entailing the responsibility of the surgeon, according to jurisprudence and medical law. Particular attention should therefore be paid to the use of compresses and surgical drapes during surgical procedures.

\section{REFERENCES}

1. O'Connor AR, Coakley FV, Meng MV, Eberhardt SC. Imaging of retained surgical sponges in the abdomen and pelvis. AJR Am J Roentgenol. 2003; 180: 481-9. 
2. Erguibi D, RH Farah, Ajbal M, Kadiri B. Textilome abdominal à propos d'un cas. Pan African Medical Journal. 2015; 21: 244.

3. Yildirim S, Tarim A, Nursal, TZ. «Éponge chirurgicale conservée (gossypibome) après une chirurgie intra-abdominale ou rétropéritonéale: 14 cas traités dans un seul centre», Langenbeck's Archives of Surgery. 2006; 391, 4: 390-395.

4. Kalovidouris A, Kehagias D, Moulopoulos L, Gouliamos A, Pentea S, et L. Vlahos, «Éponges chirurgicales abdominales retenues: aspect CT», European Radiology. 9(7): 1407-1410, 1999.

5. Wan W, Le T, Riskin L et A. Macario, «Améliorer la sécurité dans la salle d'opération: une revue systématique de la littérature des éponges chirurgicales retenues», Current Opinion in Anaesthesiology.2009; 22(2): 207-214.

6. S. Gupta et AK Mathur, «Migration transmurale spontanée de l'éponge chirurgicale provoquant une petite obstruction intenstine et une grande obstruction intestinale», ANZ Journal of Surgery. 2010; 80(10), 756-757.

7. Hammoud D, Ammouri N, Rouhana G, Saad H, Husseini H, Abou Sleiman C, Haddad M. Aspects radiologiques des textilomes. Journal de radiologie. 2001;82(8):913-6.

8. Boutayeb A, Marmade L, Laaroussi M, Bensouda A, Moughil S. Textiloma mimicking a pericardial hydatid cyst: a case report. Heart, Lung and Circulation. 2012 May 1;21(5):278-80.

9. Marchiori E, Zanetti G, Hochhegger B. Hydatid disease versus textiloma: a diagnostic challenge Thorax. 2011; 66:635.

10. Cattaneo F, Graffeo M, Brunetti E. Extrahepatic textiloma long misdiagnosed as calcified echinococcal cyst. Case Rep Gastrointest Med. 2013; 2013:261685. 